\title{
A New Ionosphere Monitoring Service over the ASG-EUPOS Network Stations
}

\author{
Anna Krypiak-Gregorczyk, Pawel Wielgosz, Marta Krukowska \\ Institute of Geodesy, University of Warmia and Mazury in Olsztyn, Oczapowskiego 1 st., 10-719, Olsztyn, Poland
}

\begin{abstract}
The paper presents the structure, performance and preliminary results of new ionosphere monitoring service over the ASG-EUPOS network of GNSS reference stations in Poland. This dense permanent network consisting of $\sim 110$ stations is a part of the European Position Determination System (EUPOS) project involving 18 countries of Central and Eastern Europe. The service was developed at the University of Warmia and Mazury in Olsztyn and as one of few uses the undifferenced carrier phase measurements from dual-frequency receivers for modeling of the ionosphere. It provides ionosphere maps (TEC) with spatial resolution of $0.25 \times 0.25$ degrees and with temporal resolution of 5 minutes. In addition, the service provides daily and monthly differential code bias (DCB) values for each of the GNSS receivers of the ASG-EUPOS system. The DCBs are estimated based on phase-smoothed pseudoranges.
\end{abstract}

Keywords: ionosphere; TEC; ionospheric delay; GNSS.

\begin{tabular}{|ll|}
\hline Nomenclature \\
$L 4_{i}^{k} \quad$ geometry-free linear combination \\
$L 1_{i}^{k} \quad$ L1 carrier phase observable \\
$L 2_{i}^{k} \quad$ L2 carrier phase observable \\
$\Delta I_{i}^{k} \quad$ ionospheric delay \\
$B_{i 4}^{k} \quad$ ambiguity parameter \\
Greek symbols \\
$\xi_{4} \quad$ conversion parameter for ionospheric delay \\
\hline
\end{tabular}

\section{Introduction}

The ionosphere region plays an active and important role in the complex space weather relationships, in ground-based and satellite radio communication and navigation, and therefore affects important geodetic systems (e.g., Global Navigation Satellite Systems - GNSS). So permanent monitoring of the ionospheric state is required. It should be noted that though accuracy and reliability of GNSS suffer from the ionospheric impact on navigation signals, these systems offer the chance for a permanent monitoring of the Total Electron Content (TEC) up to about $20000 \mathrm{~km}$ height [22]. The possibilities to study the ionosphere have been greatly extended when satellite navigation systems were built. These systems have become some kind of ionosphere scanners and are excellent supplements of classical sounding methods that allow for modeling the ionosphere and its disturbances with unprecedented temporal and spatial coverage. Earlier, the ionosondes were the principal source of the ionosphere sounding, but their number and information delivery only related to the conditions in the lower part of ionosphere over limited areas limited their suitability for generating accurate regional or global ionosphere models [11]. Nowadays, determination of ionospheric TEC in regional and global scales relies on GNSS data from ground permanent networks (IGS, EPN, CORS, etc.). Possibilities and quality of the research on spatial and temporal changes in the ionosphere are growing with rising both the number of permanent GNSS stations and the number of GNSS systems (and therefore the number of available satellites).

The ionosphere is characterized by very dynamic changes not only during magnetic storms or adverse solar activity events (solar flares, coronal mass ejections) [2]. Thereby in climatological research on the ionosphere, the accuracy and

Corresponding author: Pawel Wielgosz. E-mail address: p.a.wielgosz@gmail.com

http://dx.doi.org/10.3846/enviro.2014.224

(C) 2014 The Authors. Published by VGTU Press. This is an open-access article distributed under the terms of the Creative Commons Attribution License, which permits unrestricted use, distribution, and reproduction in any medium, provided the original author and source are credited. 
resolution offered by the current global and regional models is often not satisfactory. It should be noted that the accurate knowledge of the state of the ionosphere plays a key role in determining a position using GNSS data. It is well known that the reliable modeling of the ionospheric and tropospheric propagation errors is one of the most challenging aspects of precise GNSS positioning and GNSS-based geodetic and geodynamic studies [5], [10], [20]. This is due to the decisive influence of the ionospheric delays on the possibility of determining the carrier phase ambiguities. Also, precise GNSS positioning requires accurate ionosphere models to support carrier phase ambiguity resolution. Therefore, the development of high accuracy models with higher spatial and temporal resolution is required [4], [18].

Currently there are various global, regional and local ionosphere models used in GNSS data processing and ionospheric research. The most of the ionosphere models are based on carrier phase-smoothed pseudorange data, which presents low accuracy and requires strong smoothing of the results. The ionospheric delay obtained from smoothed pseudoranges have accuracy of about a few TECU. This is one of the reasons why most of the research centers use spherical harmonics expansion for TEC parameterization in their global and regional solutions [12-13]. As a result, the obtained ionosphere maps are characterized by low spatial resolution of few degrees and temporal resolution of 5 do 120 minutes. Two of the most popular of the ionosphere models are that provided by the International GNSS Service (IGS) and the Center for Orbit Determination in Europe (CODE). The IGS global ionospheric maps (GIMs) are a combination of several independent solutions provided by the IGS Analysis Centers [6]. This global model uses $\sim 200$ permanently tracking GNSS stations and CODE GIMs are computed using data from about 150 GNSS sites of the IGS. Broadly used IGS model has spatial resolution of $5.0 \times 2.5$ degrees and temporal resolution of 2 hours. Also, its estimated accuracy is about 4 TEC [6-7]. The CODE GIMs offering similar temporal and spatial resolutions to the IGS product [12]. Therefore, there is still a room for improvement in the ionosphere modelling, and the presented ionosphere monitoring service is a such attempt.

\section{Methodology}

The new ionosphere monitoring service over the ASG-EUPOS network of GNSS reference stations was developed at the University of Warmia and Mazury in Olsztyn. For the ionospheric modeling, dual-frequency GPS data from Polish national ground based augmentation system (GBAS) - ASG-EUPOS - were used. This Polish GPS network operating since 2008, gave new possibilities for accurate regional modeling of Earth's upper atmosphere [1]. This dense permanent network consisting of $\sim 110$ stations with mean distance between stations of $70 \mathrm{~km}$ is a part of the European Position Determination System (EUPOS) project involving 18 countries of Central and Eastern Europe.

The presented regional ionospheric model is computed using only precise, absolute (undifferenced) carrier phase GNSS measurements, a few order of magnitude more precise comparing to pseudorange measurements. This is an important novelty, because the previously developed regional and global ionospheric models are mostly based on carrier phasesmoothed pseudoranges and offer limited accuracy at the level of 3-4 TECU [7].

Our system computes the ionospheric delay using a geometry-free linear combination (L4) of dual-frequency carrierphase observations:

$$
\begin{gathered}
L 4_{i}^{k}=L 1_{i}^{k}-L 2_{i}^{k} \\
L 4_{i}^{k}=-\xi_{4} I_{i}^{k}+B_{i 4}^{k} \\
I_{i}^{k}=\frac{B_{i 4}^{k}-L 4_{i}^{k}}{\xi_{4}}
\end{gathered}
$$

where:

$L 4_{i}^{k}-$ geometry-free linear combination,

$L 1_{i}^{k}, L 2_{i}^{k}-\mathrm{L} 1$ and $\mathrm{L} 1$ carrier phase observations,

$\xi_{4}=1-\xi=1-\frac{v_{1}^{2}}{v_{2}^{2}} \approx-0.647-$ a factor that converts the ionospheric delay on $L 4$ to that on $L 1$,

$I_{i}^{k}-$ ionospheric delay.

An ambiguity parameter $B_{i 4}^{k}$ has undefined wavelength, therefore it is expressed in units of length:

$$
B_{i 4}^{k}=\lambda_{1} N_{i 1}^{k}-\lambda_{2} N_{i 2}^{k}-\left(b_{L 1}^{k}-b_{L 2}^{k}\right)-\left(b_{L 1, i}-b_{L 2, i}\right)
$$

It should be noted, that $L 4$ combination eliminates the geometry-related errors - clock errors of the receivers and GNSS satellites and the tropospheric delay [21]. Unfortunately, this combination includes, except information about the ionospheric delays, also carrier phase ambiguities and carrier phase hardware delays. In our study we made an attempt to 
model these parameters. For this purpose, new TEC parameterization algorithms based on L4 data and two-dimensional functions (local polynomials), was implemented. Parameter estimation was based on Least Squares Adjustment (LSA). In the first step of the data processing the ambiguity parameter for each observation arc is estimated together with parameters of the local polynomials. Once the ambiguity parameters are know it is possible to calculate precise TEC values at the ionospheric pierce points (IPP) using L4 observations. In order to represent the ionospheric vertical TEC (vTEC) at IPP locations, a single layer model (SLM) mapping function was used [12], [14].

Figure 1 illustrates locations of all ASG-EUPOS and neighboring EPN stations and figure 2 shows examples of IPP locations for their measurements collected at 06.10 UT on September $20^{\text {th }}, 2013$. As one can see, the IPP locations have a clustered distribution and are representative for other epochs over 24-hour period.

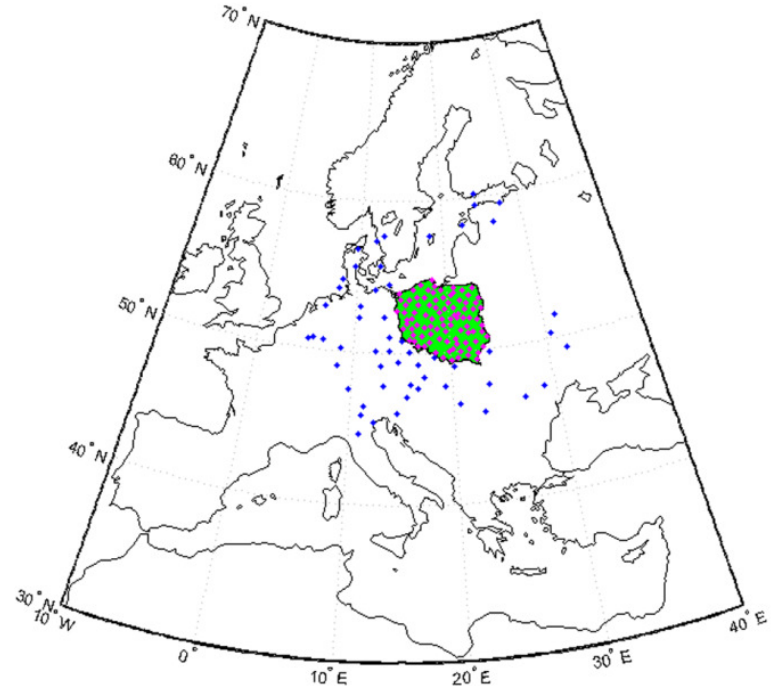

Fig. 1. Locations of ASG-EUPOS stations (red) and neighboring EPN stations (blue)

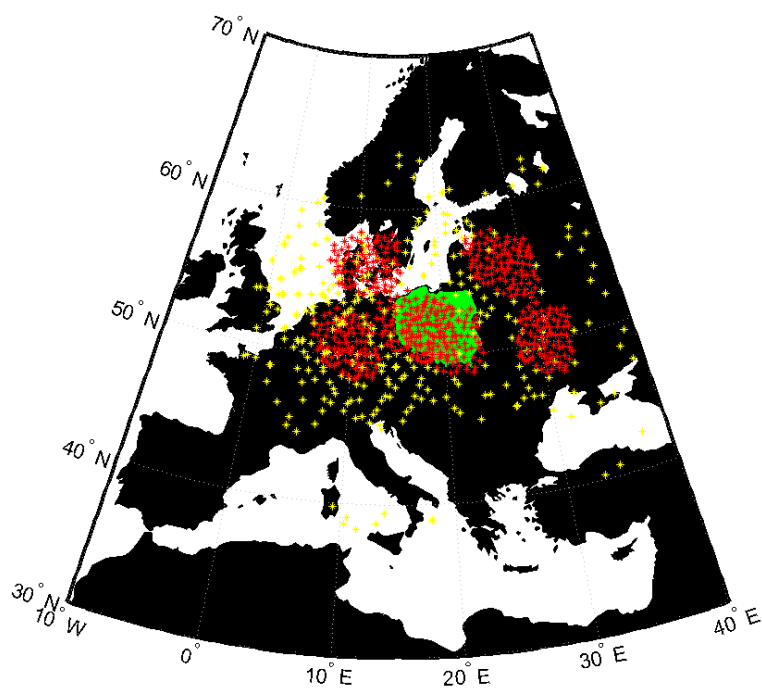

Fig. 2. Examples of IPPs locations at 06.10 UT on September 20th, 2013 (red - IPPs for ASG-EUPOS stations, yellow - IPPs for EPN)

Earlier research on the ionosphere modeling from GNSS data pointed out that interpolation methods may give better results, as compared to the functional representation of TEC using, e.g., spherical harmonics expansion [3], [9], [15-17]. Therefore the new ionosphere monitoring system uses interpolation methods based on two-dimensional local polynomials. It should be also noted that vertical TEC values estimated by using precise carrier phase observations have better accuracy than offered by standard methods based on smoothed pseudoranges. Hence, there was no need to use the functions with strong smoothing properties.

\section{Service data flow}

The data flow in the service is presented in Figure 3. First, the ASG-EUPOS and EPN RINEX data files from $\sim 150$ station are automatically downloaded through ftp protocol. Then the data import module reads data files and imports dualfrequency pseudorange and carrier phase observations which are later stored in internal binary format. Next, two parallel streems are activated, one for carrier phase observations and one for pseudorange ones. In case of the pseudorange observations respective preprocessing module checks quality of the data, next satellite elevations and azimuths with respect to all the processed stations are calculated. Also, pseudorange data is smoothed using carrier phase observations and geometry-free linear combination of the smoothed pseudoranges (P4) is created. Next, the P4 data is fed into DCB calibration module that estimates station and satellite daily DCBs. The estimated DCBs are published on the service web page.

In the meantime, carrier phase preprocessing module is executed. It detects outliers in carrier phase data and detects cycle slips resulting in clean arcs of continuous carrier phase data for every satellite. Then, geometry-free linear combination of the carrier phase data (L4) is created. The L4 data together with the calculated earlier satellite elevations and azimuths is fed into TEC mapping module. Finally, TEC maps and animations are derived and published on the service web page.

\section{Products of new ionosphere monitoring service}

Ionosphere and space weather monitoring and the derivation of relevant information concerning the structure of the ionosphere and its dynamics may be helpful for users in navigation, positioning and surveying tasks. The new ionosphere monitoring service provides ionosphere maps (TEC) and mean TEC over Poland. In addition, the service provides daily and monthly differential code bias (DCB) calibrations for every GNSS receiver of the ASG-EUPOS system.

The main product of the service is new precise local ionospheric model over ASG-EUPOS network stations based on undifferenced dual-frequency carrier-phase GNSS data. This developed model has spatial resolution of $0.25 \times 0.25$ degrees 
and temporal resolution of 5 minutes. Figure 4 presents examples of TEC maps at the selected epochs for a quiet ionosphere day (November $25^{\text {th }}, 2013$ ) In addition, Figure 5 shows TEC maps for an active ionosphere day (November $9^{\text {th }}, 2013$ ). The maps show clear effect of the ionospheric disturbances.

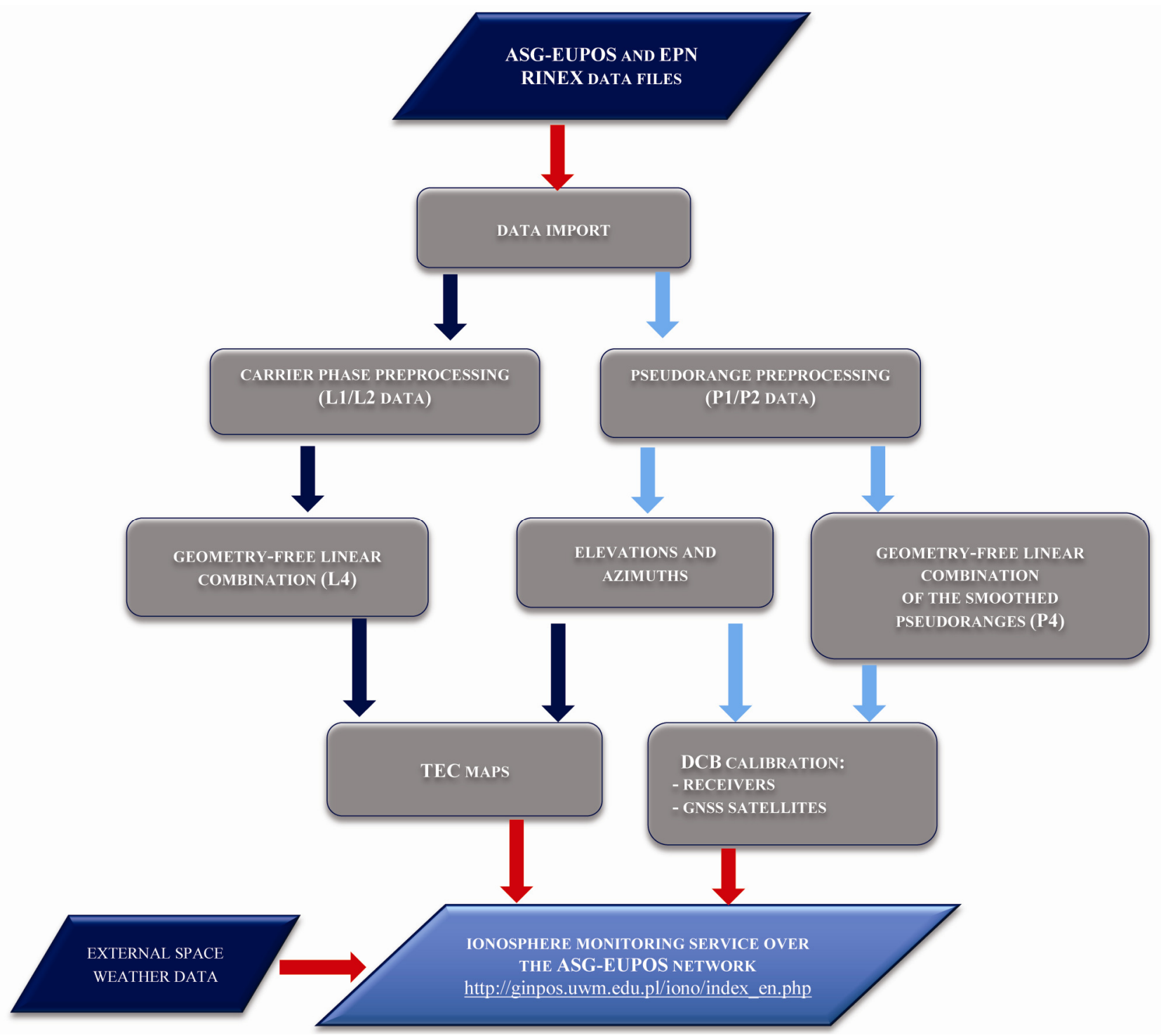

Fig. 3. Data flow in the ionosphere monitoring service over the ASG-EUPOS network

Based on the ionosphere model mean TEC values over Poland are computed every hour. This is a very important product of this service, providing the user general information about the state of the ionosphere over Poland. The user can analyze the state of the ionosphere during extended time period. Figure 5 shows examples of the mean TEC values for the example two subsequent days.
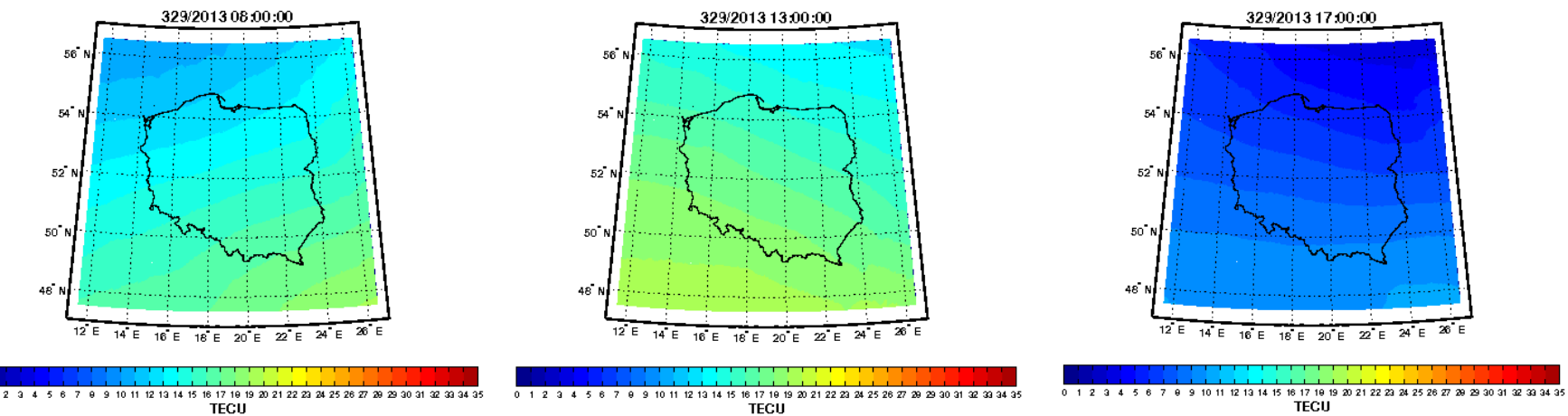

Fig. 4. Examples of TEC maps at the selected epochs for a quiet ionosphere day (November $25^{\text {th }}, 2013$ ) 

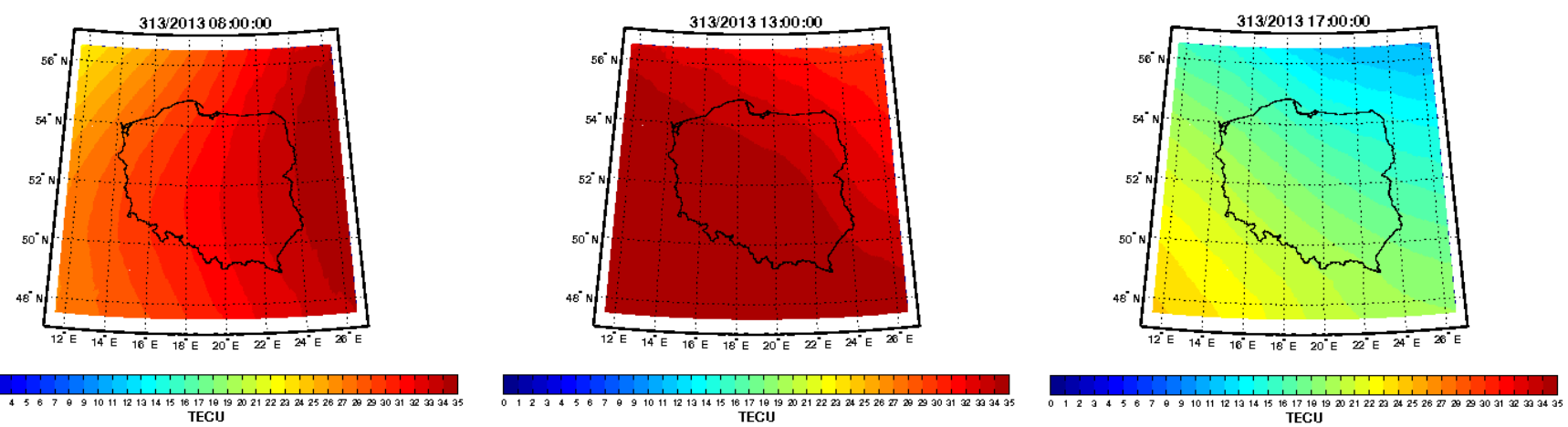

Fig. 5. Examples of TEC maps at the selected epochs for an active ionosphere day (November $\left.9^{\text {th }}, 2013\right)$
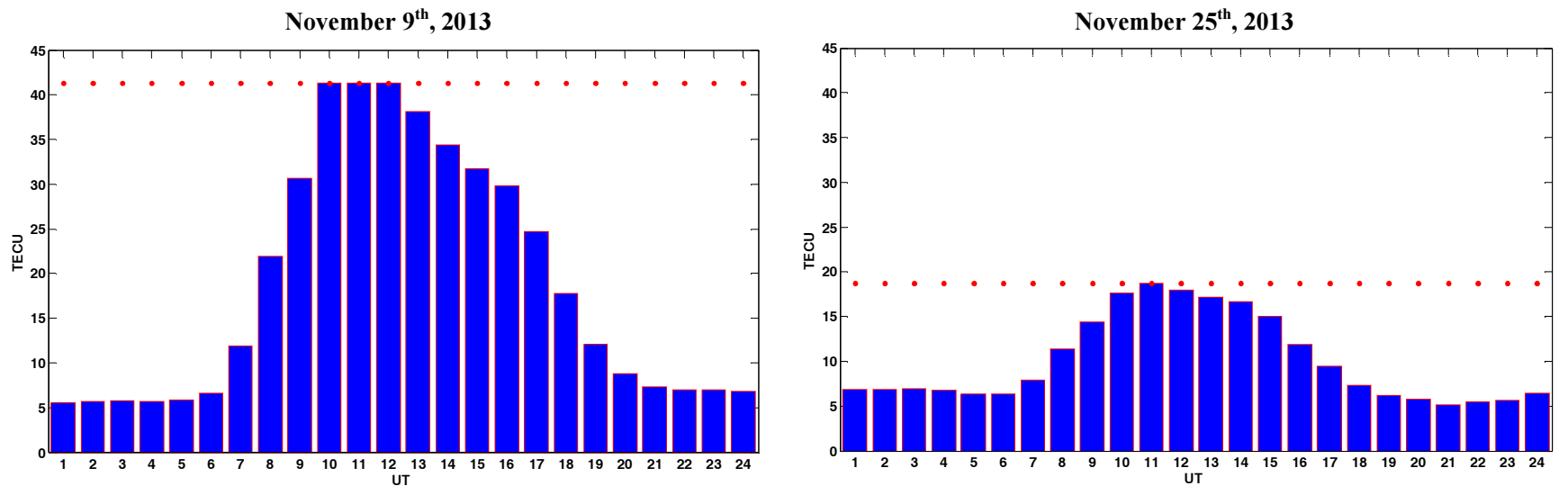

Fig. 6. Examples of mean TEC over Poland for an active (left panel) and a quiet (right panel) ionosphere days

The another important products of the new ionosphere monitoring service are the differential code biases (DCBs). It is well known that DCBs are one of the important error sources in estimating TEC from GNSS pseudorange data. DCBs for GNSS satellites and EPN ground receivers are provided by CODE Analysis Center. However, DCBs for regional and local network receivers are not provided. Therefore, our research group estimates GNSS satellite and receiver DCBs for Polish network. In this study, the ionospheric delay was computed using a geometry-free (P4) linear combination of carrier-phase smoothed pseudorange data [17]. The differential code biases were estimated for each station and satellite using the dualfrequency GPS measurements collected during a 24-hour period.

In case of EPN station used in our model, the obtained DCBs were tested and compared to the CODE reference DCBs in order to verify their accuracy. GNSS satellite DCBs and receiver DCBs for ASG-EUPOS network and selected EPN stations were calculated for all days of March 2012. The test results show a good agreement with monthly CODE DCBs. In case of receiver DCBs, mean differences between the estimated DCBs and DCBs provided by CODE (mDCB $\left.\mathrm{mSG}_{\mathrm{ASODE}}\right)$ do not exceed 0,60 ns with average standard deviation of the differences (STD) not exceeding 0,20 ns. While mean difference of satellites DCBs is equal $0,11 \mathrm{~s}$ with average standard deviation lower than $0,10 \mathrm{~ns}$ and (Table 1).

Table 1. Statistics of the comparison of the obtained DCBs with DCBs provided by CODE

\begin{tabular}{lll}
\cline { 2 - 3 } & DCBs \\
\cline { 2 - 3 } & for EPN ground receivers [ns] & for GNSS satellites [ns] \\
\hline mDCB $_{\text {ASG-CODE }}$ & 0,54 & 0,11 \\
\hline STD & 0,17 & 0,09 \\
\hline
\end{tabular}

It is well known that the total electron content displays primarily day-to-night variations, but also depends on the geomagnetic latitude, time of year, and the sunspot number [19]. Therefore, presented service provides not only the developed ionospheric products but also the information of current space weather. 


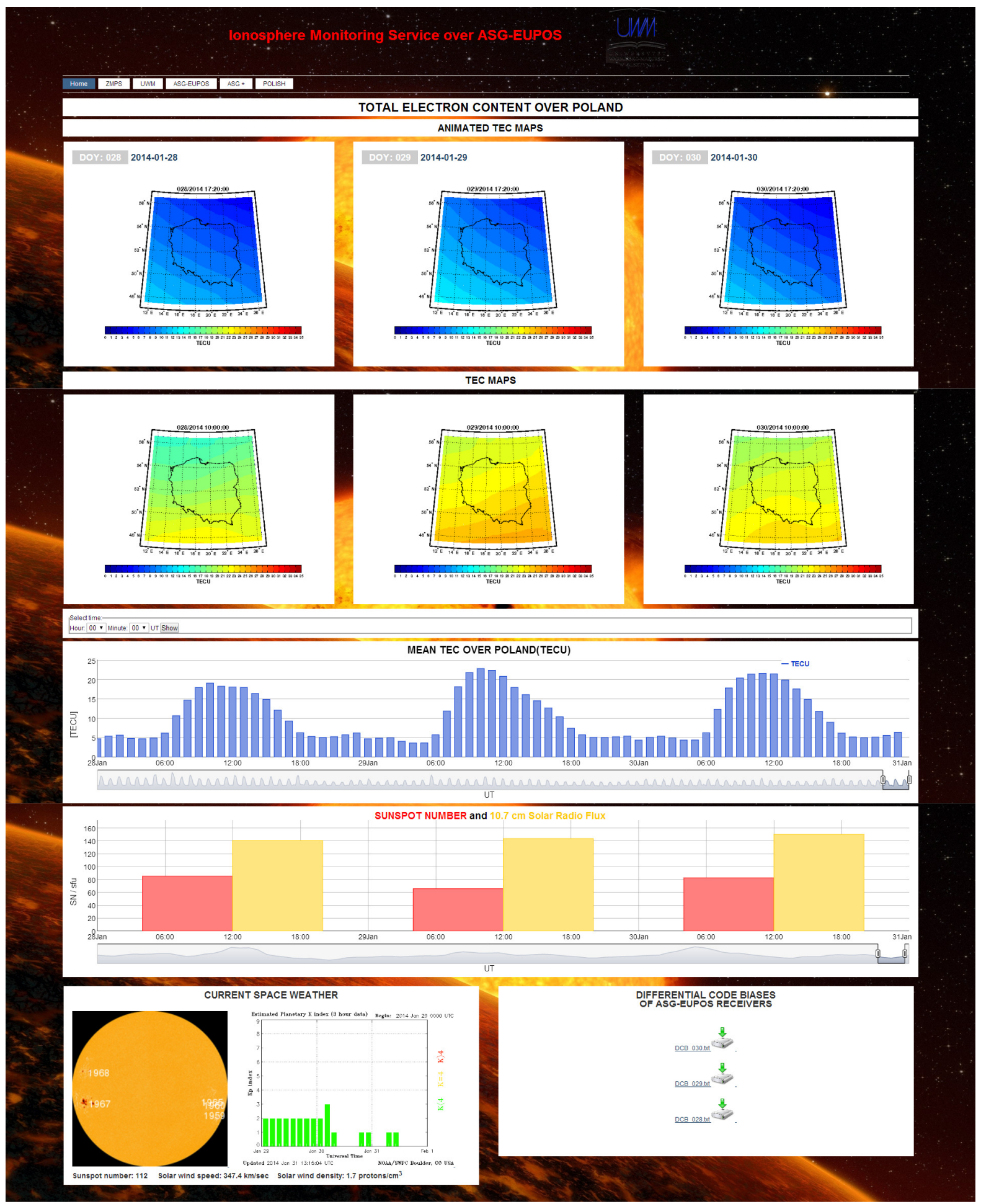

Fig. 7. The layout of the service web page (http://ginpos.uwm.edu.pl/iono/index_en.php)

It has been shown by Davies [21] that TEC is very sensitive to variations in the solar radiation. There is a close relationship between solar space weather information and the corresponding ionospheric response. The informations of current space weather are delivered in the form of products offered by other space weather services. Comparison of sunspot number, solar radio flux index, geomagnetic index Kp with mean TEC could be of interest for GNSS users. It should be noted that ionospheric changes of the total ionization are slightly delayed against the variation of the $10.7 \mathrm{~cm}$ solar radio flux [8]. Our service used space weather parameters provided by the NOAA Space Environment Center. The layout of the service web page is given in Figure 7. 


\section{Conclusions}

The paper presents our preliminary results of the new ionosphere monitoring service over the ASG-EUPOS network. It provides the users important information on the state of the ionosphere and also space weather. What is important, the new service is also dedicated to the scientific community as full understanding and modeling of physical processes that occur on the Earth's upper atmosphere and the development of methods taking into account their impact on the operation of the radio systems (e.g., GNSS) is an extremely important issue, showing many open research problems. Thus the presented open service may contribute to development of geodesy and satellite navigation, and also geodynamic and geophysical studies.

\section{Acknowledgements}

This research was supported by grant no. NR09-0010-10/2010 from National Centre for Research and Development.

\section{References}

[1] Bosy, J.; Oruba, A.; Graszka, W.; Leończyk, M.; Ryczywolski, M. 2008. ASG-EUPOS densification of EUREF permanent network on the territory of Poland, Reports on Geodesy 2(85): 105-112.

[2] Brunini, C.; Van, Zele M.A.; Meza, A.; Gende, M. 2003. Quiet and perturbed ionospheric representation according to the electron content from GPS signals, J Geophys Res 108(A2): 1056. http://dx.doi.org/10.1029/2002JA009346

[3] Gao, Y.; Liao, X.; Liu, Z. Z. 2002. Ionosphere Modeling Using Carrier Smoothed Ionosphere Observations from a Regional GPS Network, Geomatica 56(2): 97-106.

[4] Grejner-Brzezinska, D. A.; Wielgosz, P.; Kashani, I.; Smith, D. A.; Robertson, D. S.; Mader, G. L.; Komjathy, A. 2006. The impact of severe ionospheric conditions on the accuracy of RTK position estimation: performance analysis of various ionospheric modeling techniques, Navigation 53(3): 203-217.

[5] Grejner-Brzezinska, D. A.; Arlsan, N.; Wielgosz, P.; Hong, C.-K. 2009. Network Calibration for Unfavorable Reference-Rover Geometry in NetworkBased RTK: Ohio CORS Case Study, Journal of Surveying Engineering 135(3): 90-100. http://dx.doi.org/10.1061/(ASCE)0733-9453(2009)135:3(90)

[6] Hernández-Pajares, M.; Juan, J. M.; Sanz, J.; Orus, R.; Garcia-Rigo, A.; Feltens, J.; Komjathy, A.; Schaer, S.; Krankowski, A. 2009. The IGS VTEC Maps: A Reliable Source Of Ionospheric Information Since 1998, Journal of Geodesy 83(3-4): 263-275. http://dx.doi.org/10.1007/s00190-008-0266-1

[7] Hernandez-Pajares, M.; Juan, J. M.; Sanz, J.; Aragon-Angel, A.; Garcia-Rigo, A.; Salazar, D.; Escudero, M. 2011. The ionosphere: effects, GPS modeling and the benefits for space geodetic techniques, Journal of Geodesy 85(12): 887-907. http://dx.doi.org/10.1007/s00190-011-0508-5

[8] Jakowski, N.; Fichtelmann, B.; Jungstand, A. 1991. Solar activity control of ionospheric and thermospheric processes, J. Atmos. Terr. Phys. 53: 11251130. http://dx.doi.org/10.1016/0021-9169(91)90061-B

[9] Krypiak-Gregorczyk, A.; Wielgosz, P.; Gosciewski, D.; Paziewski, J. 2013. Validation of approximation techniques for local total electron content mapping, Acta Geodynamica et Geomaterialia 10(3): 275-283. http://dx.doi.org/10.13168/AGG.2013.0027

[10]Paziewski, J.; Wielgosz, P. 2013. Assessment of GPS + Galileo and multi-frequency Galileo single-epoch precise positioning with network corrections, GPS Solutions. http://dx.doi.org/10.1007/s10291-013-0355-3

[11]Reinisch, B. W.; Huang, X.; Galkin, I. A.; Paznukhov, V.; Kozlov, A. 2005. Recent advances in real-time analysis of ionograms and ionospheric drift measurements with digisondes, J. Atmos. and Solar-Terr. Phys. (67): 1054-1062. http://dx.doi.org/10.1016/j.jastp.2005.01.009

[12] Schaer, S. 1999. Mapping and Predicting the Earth's Ionosphere Using the Global Positioning System. PhD Thesis, Astronomical Institute, University of Berne.

[13]Schmidt, M.; Dettmering, D.; Moßmer, M.; Wang, Y.; Zhang, J. 2011. Comparison of spherical harmonic and B spline models for the vertical total electron content, Radio Science 46, RS0D11. http://dx.doi.org/10.1029/2010RS004609

[14] Shagimuratov, I. I.; Baran, L. W.; Wielgosz, P.; Yakimova, G. A. 2002. The structure of mid- and high-latitude ionosphere during September 1999 storm event obtained from GPS observations, Annales Geophysicae 20(6): 665-671.

[15] Stanislawska, I.; Juchnikowsi, G.; Cander, Lj. R. 1996. The kriging method of ionospheric parameter f0F2 instantaneous mapping, In annali di Geofisica 39(39).

[16] Stanislawska, I.; Juchnikowsi, G.; Cander, Lj. R.; Ciraolo, L.; Bradley, P. A.; Zbyszynski, Z.; Swiatek, A. 2002. The kriging method of TEC instantaneous mapping, Adv. Space Res. 29(6): 945-948. http://dx.doi.org/10.1016/S0273-1177(02)00050-9

[17] Wielgosz, P.; Grejner-Brzezinska, D. A.; Kashani, I. 2003. Regional ionosphere mapping with kriging and multiquadric methods, Journal of Global Positioning Systems 2(1): 48-55. http://dx.doi.org/10.5081/jgps.2.1.48

[18] Wielgosz, P.; Kashani, I.; Grejner-Brzezinska, D. A. 2005. Analysis of Long-Range Network RTK during Severe Ionospheric Storm, Journal of Geodesy 79(9): 524-531. http://dx.doi.org/10.1007/s00190-005-0003-y

[19]Zakharenkova, I. E.; Krankowski, A.; Shagimuratov, I. I.; Cherniak, Yu.V.; Krypiak-Gregorczyk, A.; Wielgosz, P.; Lagovsky, A. F. 2012. Observation of the ionospheric storm of October 11, 2008 using FORMOSAT-3/COSMIC data, Earth, Planets and Space 64(6): 505-512. http://dx.doi.org/10.5047/eps.2011.06.046

[20]Böhm, J.; Schuh, H. 2013. Atmospheric Effects in Space Geodesy. Springer, Inc. ISBN 978-3-642-36932-2. http://dx.doi.org/10.1007/978-3-642$36932-2$

[21]Davies, K. 1990. Ionospheric Radio. Peter Peregrinus, London. http://dx.doi.org/10.1049/PBEW031E

[22] Jakowski, N.; Wehrenpfennig, A.; Heise, S.; Schlüter, S.; Noack, T. 2001. Space Weather Effects in the Ionosphere and their impact on positioning, in Proc. of Space weather workshop, ESTEC. 